\title{
In ricordo del professor David Romano
}

David Romano è venuto a mancarci il 12 ottobre 2001, ma la sua figura rimane viva attraverso gli scritti. Noi, colleghi e discepoli suoi, legati in vario modo alla cattedra di Filologia Italiana dell'Universitat de Barcelona di cui fu il primo titolare, vogliamo risentire la sua voce rendendo nel contempo omaggio alla sua persona.

Ci è sembrato a tale fine opportuno ricuperare un suo magistrale saggio sul codice catalano dei Trionfi di Petrarca apparso sul «Boletín de la Real Academia de Buenas Letras de Barcelona» (tomo XLI, 1987-1988, p. 5-18): un contributo ammirevole per rigore metodologico e chiarezza espositiva che brillantemente si riallaccia alla migliore tradizione dell'italianismo catalano.

Ringraziamo Mercedes Romano e la Acadèmia de Bones Lletres per l'autorizzazione generosamente concessa a ripubblicare il saggio sulla nostra giovane rivista.

María de las Nieves Muñiz Muñiz A nome della Redazione dei Quaderns d'Italià e dei colleghi italianisti operanti in Catalogna 